\title{
PWIS: Personalized Web Image Search using One-Click Method
}

\author{
Pushpa C.N. \\ Department of Computer \\ Science and Engineering \\ University Visvesvaraya \\ College of Engineering \\ Bangalore University, \\ Bangalore-560001
}

\author{
Vinay Kumar N.K. \\ Department of Computer \\ Science and Engineering \\ University Visvesvaraya \\ College of Engineering \\ Bangalore University, \\ Bangalore-560001
}

\author{
Thriveni J. \\ Department of Computer \\ Science and Engineering \\ University Visvesvaraya \\ College of Engineering \\ Bangalore University, \\ Bangalore-560001
}

\author{
Venugopal K.R. \\ Department of Computer \\ Science and Engineering \\ University Visvesvaraya \\ College of Engineering \\ Bangalore University, \\ Bangalore-560001
}

\begin{abstract}
Personalized Web Image search is the one searching for the particular images of User intention on the Web. For searching images, a user might provide query terms like keyword, image file, or click on few image file, and therefore the system can determine the images similar to the query. The similarity used for search criteria could be Meta tags, color distribution in images, region/shapes attributes, etc. Web-scale image search engines namely Google and Bing searches for images are relying on the surrounding text features. It is highly cumbersome and complicated for the web-scale based image search engines to interpret users search intention only by querying of keywords. This leads to the incorporation of noise and high ambiguity in the search results which are extremely unfit in the context of the users. It's also a necessary mandate for using visual information for solving the problem of ambiguity in the text-based image retrieval scenario. In the case of Google search, search text box will auto complete while user is typing similar added keywords. This method will differ from user intention while searching. So to avoid this kind of faults, it is important to use visual information in order to solve the uncertainty in text-based image retrieval. To retrieve exact matching, and acquire user's intention we can allow them text query with extended or related images as a suggestion. We have proposed an innovative Web image search approach. It only needs the user to click on one query image with minimal effort and images from a pool fetched by text-based search are re-ranked based on both visual and textual contents.
\end{abstract}

\section{Keywords}

Image search, Keyword Expansion, One-Click Method, Personalized Web Image Search, Re-ranking, User Intention.

\section{INTRODUCTION}

As the variety and size of digital image collections grow exponentially, efficient image retrieval is becoming more and more important. A Web image search engine is an essential aspect for web searchers to search out desired images. At present billions of images are available with the rapid growth of the Web, hence it is very difficult task for finding and accessing the image of user interest. Therefore, some additional processing is required in order to form such collections searchable

in a useful manner. The current Web image retrieval search engines, including Google image search, Bing etc., use keywords to look for images, without reviewing image content. However, when the neighboring words are ambiguous or even irrelevant to the image, the search relies on text only will result in many undesired result images.

Many commercial web image search engines still rely on keywords as queries i.e. fetch and rank images depends on neighboring text or human-submitted annotations. Users submit query keywords in the concern of finding a definite type of images. The search engine forwards thousands of images ranked by the keywords derived from the neighboring text. It is notable that text-based image search suffer from the ambiguity of query keywords. The keywords issued by users lean to be short. They cannot illustrate the content of images precisely. The search results are noisy and consist of images with quite dissimilar semantic meanings.

Motivation: A web image search engine is an essential aspect for web searchers to search out desired images. These search engines use keyword to search, user has to type keyword and search for images. The resulted search images may be ambiguous and noisy. It is difficult to achieve objective of users only by query keywords and this cause to equivocal and noisy search results which are far from user satisfactory. So, our goal is to improve the resultant search images by one click method.

Contribution: We have proposed a novel Personalized Web Image Search approach to resultant search images of user intention. It requires the user to click on one query image with minimum effort and images from a pool retrieved by textbased search are re-ranked based on both visual and textual content.

Organization: The organization of the paper is as follows: Section 2 reviews the related work of the existing works of the Image Retrieval, Section 3 explains the proposed Architecture of the System, and Section 4 presents the Problem definition and the proposed algorithm. The implementation is given in Section 5, the Performance Analysis and the results of are described in Section 6 of the paper.Finally the paper is concluded in Section 7.

\section{RELATED WORK}

Image based search has become a new feature due to availability of images on the internet which has become a factor for searching on the internet. Strategies which focus on text based search for images have drastically failed as visual image signal is ignored. Yadav et al., [1] has proposed a 
scheme in which text based search results is re-ranked based on adaptive visual symmetry. An image is queried and then categorized initially. The image specific similarity is computed which is used to re-rank and combine the images based on queried image.

Retrieval of images from large and distinct collections using image content as a key is a challenging and vital issue. The advantage of textual content based search of Web pages and based on the complexity and cost of utilizing the image based signals, several search engines return images purely rely on the text content of the pages from which the images are related. There is no image analysis to verify the quality and the relevance of the image. This provides results that are subject to a high level of inconsistency and are of low quality. So, such kind of visual search method has proven unsatisfying as it often completely ignores the visual content itself as a grading signal. To address this problem, Chouragade et al., [2] have presented a new image ranking technique known as visual re-ranking methodology, for reordering images with respect to their visual appearance. This method relies on analyzing the distribution of visual resemblance among the images and image grading system that checks the multiple visual themes and their relative strengths in a large group of images.

Winston Hsu et al., [3] have proposed an Information Bottleneck (IB) re-ranking strategy for reordering the results of text based image extraction based on approximation of relevance of text oriented extracted image patterns. Information Bottleneck (IB) principle is based on optimal image clustering technique that preserves maximum mutual information between the image search relevance and the dimensionality of images. This strategy is generic and performance is maximum for sophisticated models tuned for a class of queries narrowed down towards specific query class.

Yuchi Huang et al., [4] propose a new inductive knowledge acquisition framework for image fetch, in which images are taken as vertices in a weighted hypergraph and the venture of image probe is formulated as the problem of hypergraph priority.

Nadav Ben-Haim et al., [5] proposed the method for searching images using search engines on the web mainly based on the keywords with the name of the files and images which will get unwanted things in the search results. This system is to represent the interest on object and re-ranks the images based on their relevant content. The quality of the system is that the user should enter only a keyword to represent query in feature space to re-rank. They describe how to find a picture representation of a text query using the sets of images returned by image search engines.

R. Fergus et al., [6] extend the model of pattern to include heterogeneous parts to represent the appearance or the geometry object in the region. The cluttered images and their configuration can be done at a time automatically without supervision. Authors described the model which can be employed for ranking the output of an image search engine when searching for object based on categories. It is shown that picture consistencies in the output of the images can be identified, and then ranking the images which is closeness to the visual object category. The proportion of good images may be small, the algorithm is designed to withstand and it is capable of learning in either a totally unsupervised manner, or with a very limited amount of supervision.
Gunhan Park et al., [7] addressed a problem of ranking to retrieve web image. Now a days we get more comprehensive of web images retrieve can be expected. Conventional systems for web image can be extracted based on keyword. Authors find undesirable results from the keyword based on web image system since the system uses some amount of not accurate text information of web images and a quality of a system uses text information such as images or texts, etc. To modify this, the authors propose a new ranking scheme which is the integration of outcomes of text and image content via analyzing the results. The majority based approach, especially with the centroid-of-largest-cluster method, has better effectiveness than the initial method using only text evidence.

Web image search using text queries has received significant attention. However, current state-of-the-art methods need training models for each new query and are consequently unfit for real-world web search programs. The prime contribution of the paper [8] is introduction to generic groupings based on query-relative attributes which can be utilized for new queries without subsidiary training. They combine textual attributes, with respect to the occurrence of query terms in web pages and image meta-data, and visual histogram demonstration of images.

Chum et al. [9] used RANSAC to verify the spatial configurations of local visual features and to purify the expanded image examples. However, it was only applicable to object retrieval. It required users to draw the image region of the object to be retrieved and assumed that relevant images contained the same object.

Under the framework of pseudo relevance feedback, Ah-Pine et al. [10] proposed transmedia similarities which combined both textual and visual features. Krapac et al. [11] proposed the query-relative classifiers, which combined visual and textual information, to re-rank images retrieved by an initial text-only search. However, since users were not required to select query images, the users' intention could not be accurately captured when the semantic meanings of the query keywords had large diversity.

Besides visual query expansion, some approaches [12], [13] used concept-based query expansions through mapping textual query keywords or visual query examples to high-level semantic concepts. They needed a predefined concept lexicons whose detectors were offline learned from fixed training sets. These approaches were suitable for closed databases but not for web-based image search, since the limited number of concepts cannot cover the numerous images on the Internet. The idea of learning example specific visual similarity metric was explored in previous work [14], [15].

Huang et al. [16] proposed probabilistic hypergraph ranking under the semi-supervised learning framework. It utilized both labeled and unlabeled images in the learning procedure. Relevance feedback required more users' effort. For a webscale commercial system, users' feedback has to be limited to the minimum, such as one-click feedback.

One of the major challenges of content-based image retrieval is to learn the visual similarities which reflect the semantic relevance of images well. Image similarities can be learned from a large training set where the relevance of pairs of images is known. Deng et al. [17] learned visual similarities from a hierarchical structure defined on semantic attributes of training images. Since web images are highly diversified, 
defining a set of attributes with hierarchical relationships for them is challenging.

\subsection{Background}

Many Web scale image search strategies which extract images from the internet are text-based and have limitations owing to the fact that querying based on keywords cannot give a clear description of the image content with high accuracy. Contentbased image retrieval [18] paradigm uses visual features for evaluation of the image similarity. For the sole purpose of reducing the number of users', pseudo relevance feedback expanded the query image by taking the top $\mathrm{N}$ images that correlate to a high degree of visual similarity for querying the image as positive examples. Owing to the semantic gap, the first $\mathrm{N}$ images would not be consistent semantically with the query image. Incorporation of visual expansion features for the image querying process, the images are searched. They are in need of a pre-defined concept of lexicons whose detectors were off-line when learned from the fixed training sets. These approaches were best suited for the traditional database oriented searches but do not fit into the scope of web-based image search owing to the reason of usage of the limited number of concepts that cannot cover the large number of images on the Internet.

Keyword expansion technique is incorporated to expand the retrieved image pool and to add to the positive examples. This feature is mainly used in the scenario of document retrieval. Some algorithms generated tag based suggestions or annotations based on the visual content for input images. Their objective is not to focused to improve the performance of image re-ranking but focused on keyword expansions. Certain difficulties prevent them from being directly used to address the problem. Most of them assumed fixed keyword sets, which are tedious for image re-ranking in open and dynamic web environment.

\section{PROBLEM DEFINITION}

There are many images are stored in the database. The objectives of this work are:

1. To Capture the Web Images of User Intention using One-Click Method.

2. To improve the Precision, Recall and F-measure of the proposed System.

\section{ARCHITECTURE}

As shown in the Fig. 1, the system accepts the user query from the user, once the user enters the keyword and press enter or click go or search, the query will be passed to search engine. Search engine will query the image pool or database (based on the attributes stored for the images) and forms a cluster for the images based on the user query.

Search engine will uniquely identify the images depending on the image characteristics and retrieves only the unique characteristic images (best image) randomly from the database. Finally, the unique images related to the search will be displayed on the main screen of user.

This process is automatic and will help the user to decide about what are the search results he / she may be interested in. By looking at the unique clustered images related to the query given, the user will decide to go with one of the unique image retrieved.

Once the user clicks on the image of interest, the search engine will then expand the query using keyword expansion internally behind the scenes and collect all the images in the image pool depending on the characteristic of the image selected by the user. Finally, the collected image pool results will be displayed on the main screen of the user, this process is very simple and user feels happy of the results because of the required results with hassle free interface and the search engine does not asks for many inputs or clicks.
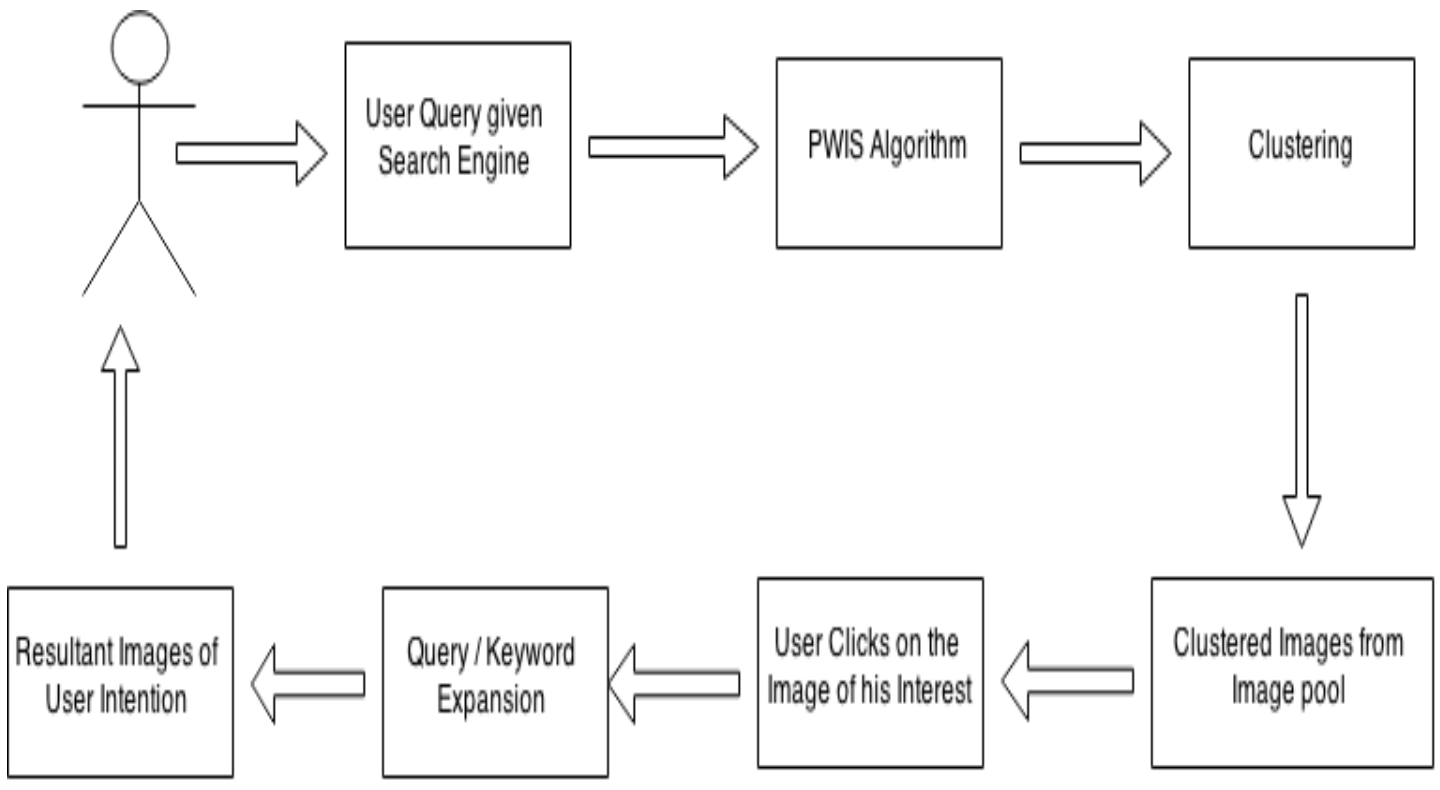

Fig. 1. System Architecture

\section{IMPLEMENTATION}

The following steps required to capture the user intention images from one-click query image are:
1. The query image is categorized into one of the predefined adaptive weight categories which reflect users' search intention at a coarse level. A specific weight schema is used to combine 
visual features adaptive to this kind of image to better re-rank the text based search result.

2. In the visual content of the query image selected by the user and through image clustering, query keywords are expanded to capture user intention.

3. To enlarge the image pool using expanded keywords to contain more relevant images.

4. The keyword expansion is used to expand the query image to multiple positive visual examples from which new query specific visual and textual similarity metrics are learned to further improve content-based image re-ranking.

\subsection{ALGORITHM}

The Table 1 gives the Personalized Web Image Search Algorithm to find the Web images of User intention by one single click method.

Table 1. Personalized Web Image Search Algorithm

Input: Initial weight $\mathrm{W}_{\mathrm{i}}$ for all query images $\mathrm{i}$ in the current intention category Iq, similarity characteristics for all query image weight $i$ and feature $f$;

Begin

Step 1: Initialize the query depending on the input received.

Step 2: Select best characteristics bt and the corresponding similarity cs for current re-ranking problem underweight $\mathrm{W}_{\mathrm{i}}$

Step 3: With repetitive corresponding similarity cs, for obtaining only the unique characteristics based on the initial weight $\mathrm{W}_{\mathrm{i}}$ which are in the best characteristics bt.

Step 4: A unique with Best Characteristics results bt results will be displayed.

Step 5: Depending on the user Click, the query will be expanded at the backend without coming to user's knowledge.

a. The best characteristics bt will be considered and corresponding similarity cs of the bt will be taken into consideration.

b. Depending on the above consideration, a collection of images will be formed.
6.

PERFORMANCE ANALYSIS

We have implemented this in Java programming language and used Eclipse as an extensible open source IDE (Integrated Development Environment) [15].

The following steps explain exactly how the proposed system works:

Step1: Administrator loads the images to database with their characteristics and the images are stored in file store for faster retrieval.

Step 2: User Queries the Search engine.

Step 3: Depending on the Search Keyword, Search engine clusters the related results and displays unique characteristic Images (one image for each characteristic) depending on the search keyword.

Step 4: User decides on the intended result and clicks on one of the cluster images

Step 5: Depending on the user intention or click, the search engine will expand the query internally.

Step 6: Search engine will collect all the related information depending on the characteristic of the image selected by the user.

Step 7: Search engine will then display the intended results back to user.

End 


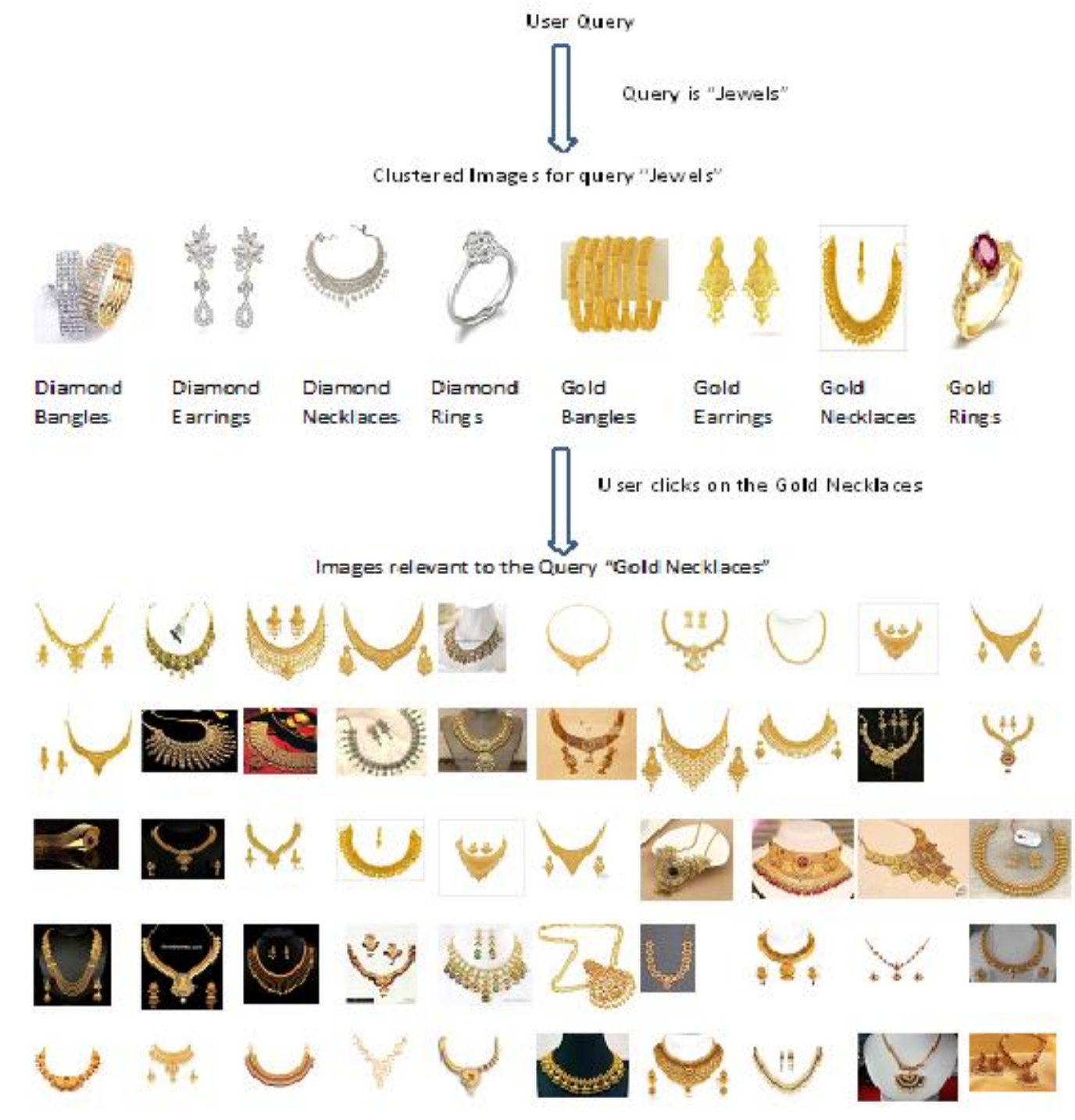

Fig. 2. Web Image Results for the Query "Jewels"

Table 2: Clustered images for 20 queries

\begin{tabular}{|c|c|}
\hline Query & Image Clusters \\
\hline Apple & Computers, Green apples, Logos, Mixed Color apples, Phones, Red apples \\
\hline Cake & Blackberry, Chocolate, Pista, Strawberry, Vanilla \\
\hline Jewels & $\begin{array}{r}\text { Diamond Bangles, Diamond Earrings, Diamond Necklaces, Diamond Rings, Gold } \\
\text { Bangles, Gold Earrings, Gold Necklaces, Gold Rings }\end{array}$ \\
\hline Teddy & Brown, Group, Heart, Pink, Purple, White \\
\hline Dell & Desktop computers, Laptop Computers, Touchscreen \\
\hline Adidas & Football, Logos, Shirts, Shoes \\
\hline Bangalore City & Buildings, Map, Metro, Road, Tourist \\
\hline Galaxy & Phones, Sky \\
\hline BPL & Campus, Computers, Logos, Products \\
\hline Wipro & Building, Logos, MOM, Rocket \\
\hline ISRO & \\
\hline
\end{tabular}




\begin{tabular}{|c|c|}
\hline Lotus & Cars, Flowers, Wallpapers, Drawings, Tattoos, Pond \\
\hline HAL & Helicopters, Medium passenger Areoplane, Light combat Aircraft \\
\hline HAL Helicopter & Dhruv /ALH, Chetek helicopter, Cheetah helicopter, Light Combat Helicopter. \\
\hline Java & Programming, Logos, Island \\
\hline Mars & Planet, Chocolate, Surface \\
\hline Solar & System energy, Water heater, Home logo, Eclipse. \\
\hline Crane & Bird, Tower, Mobile, Truck \\
\hline TATA & Motor, Truck, Logo, Bus, Ratan Tata, truck \\
\hline Net & Fishing, Nest, Texture, Internet, Network, Badminton, \\
\hline
\end{tabular}

\subsection{RESULTS}

The Fig. 2. shows an example of Web image results for the query 'Jewels'. First, User has to provide the query. If the user query is 'Jewels' then we display the clusters of Jewels such as Diamond Bangles, Diamond Earrings, Diamond Necklaces, Diamond Rings, Gold Bangles, Gold Earrings, Gold Necklaces, Gold Rings etc. with one relevant image of that particular cluster. By seeing the clustered results, the user will easily select and clicks on his intention image. As soon as he clicks on the cluster, the images of that particular cluster will be displayed. In this example, if he is interested with Gold Necklaces, then only the images of Gold Necklaces will be displayed. So, then we can achieve that, from the proposed system, the user will get images of his interest with single click. The Table 2 depicts the Image clusters for the 20 queries.
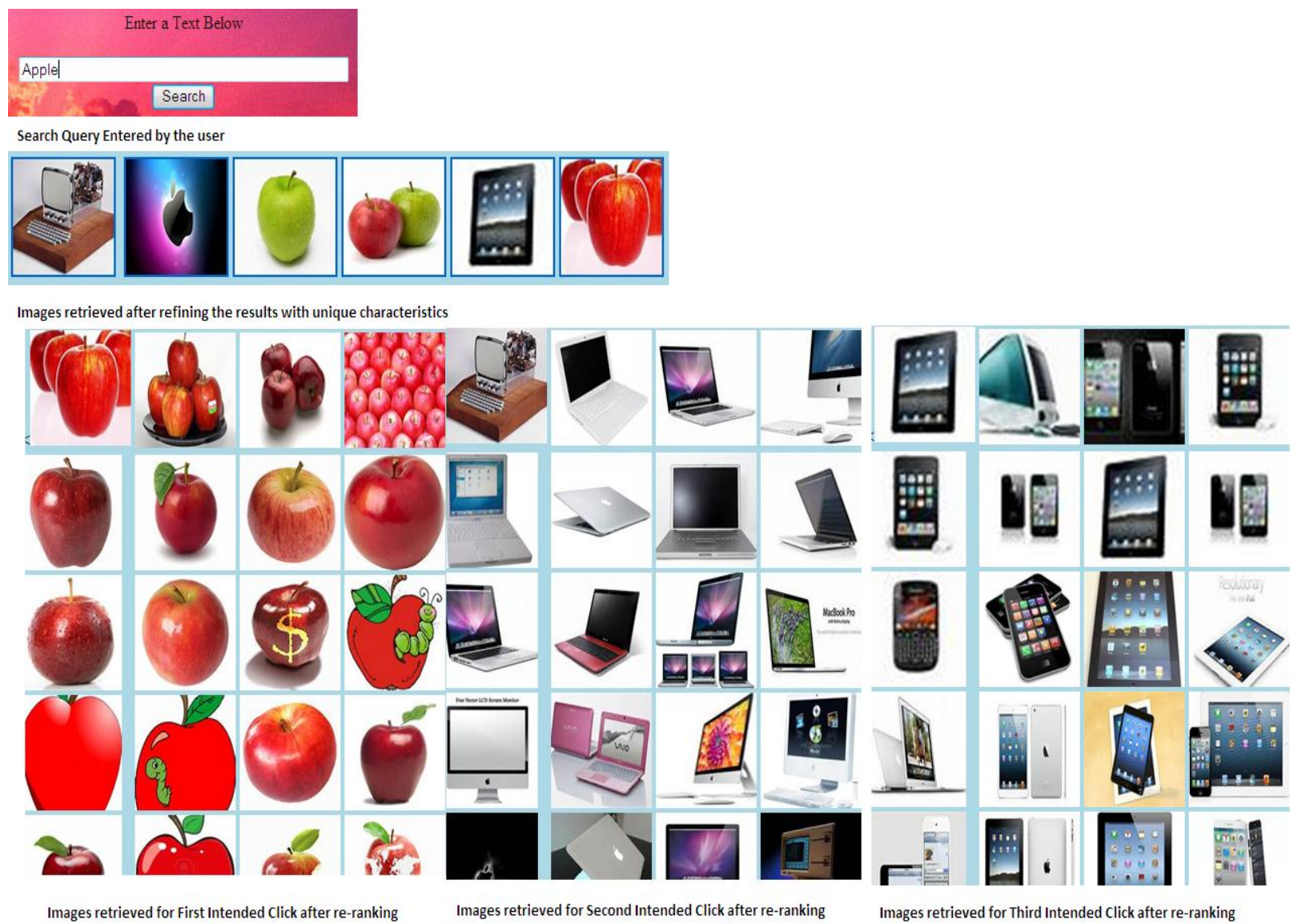

Fig. 3. Web Image Search Results for the query "Apple".

The Fig. 3 shows the Web image search results for the query "Apple". If the user enters query as Apple in the textbox, then it will displays the clusters of images of apple computer, apple 
logo, green apple, mixed apple, apple phone, red apple etc. From this clustered results, if he clicks on "red apple", then only the set of red apple images will be displayed. Similarly, if user clicks on Apple computer and apple phone, the respective cluster images will be displayed.

Fifty query keywords are chosen for evaluation of the system and using each keyword as query, the top 1,000 images are crawled from Bing image search. There are 5,000 Web images are manually labeled into different classes and stored in the database and this will be considered as training dataset. For example, for query apple, its images are labeled as red apple, apple ipod, apple computer, etc.

In this work, we have assumed that an image captures user intention when it is both semantically and visually similar to the query image. However, in some cases user intention cannot by well expressed by a single query image. For instance, the user may be interested in only part of the image.

In those cases, more user interactions, such as labeling the regions that the user thinks are important, have to be allowed.

The Precision is defined as

Precision, $\mathrm{P}=$ Number of Images retrieved that are relevant Total Number of Images those are relevant

Recall, $\mathrm{R} \equiv$ Number of Images retrieved which are relevant Total Number of Images that are retrieved

The F-Measure $\mathrm{F}$ is depicted as the harmonic mean of the Precision and the Recall R.

$$
\mathrm{F}=\frac{2 \mathrm{x} \text { Precision } \mathrm{x} \text { Recall }}{\text { Precision }+ \text { Recall }}
$$

Table 3: Performance Analysis of PWIS Algorithm

\begin{tabular}{|l|l|l|l|}
\hline Query & Precision (\%) & Recall (\%) & $\begin{array}{l}\text { F-Measure } \\
(\mathbf{\%})\end{array}$ \\
\hline Apple & 93.8 & 97.8 & 95.7 \\
\hline Cake & 97.4 & 95 & 96.2 \\
\hline Python & 92.6 & 96.7 & 94.6 \\
\hline Jewels & 98.5 & 97 & 97.7 \\
\hline Java & 90 & 90 & 90 \\
\hline Average & $\mathbf{9 4 . 5}$ & $\mathbf{9 5 . 3}$ & $\mathbf{9 4 . 8}$ \\
\hline
\end{tabular}

Table 3 shows the precision, recall and F-measure of PWIS method. As a sample, we have considered only five image queries which are mentioned in the Table 3 to calculate the Precision, recall and F-measure. The average of precision, recall and F-measure is calculated which is represented graphically in the Fig. 4 and the average performance measures of Personalized Web Image System is depicted in the Fig. 5.

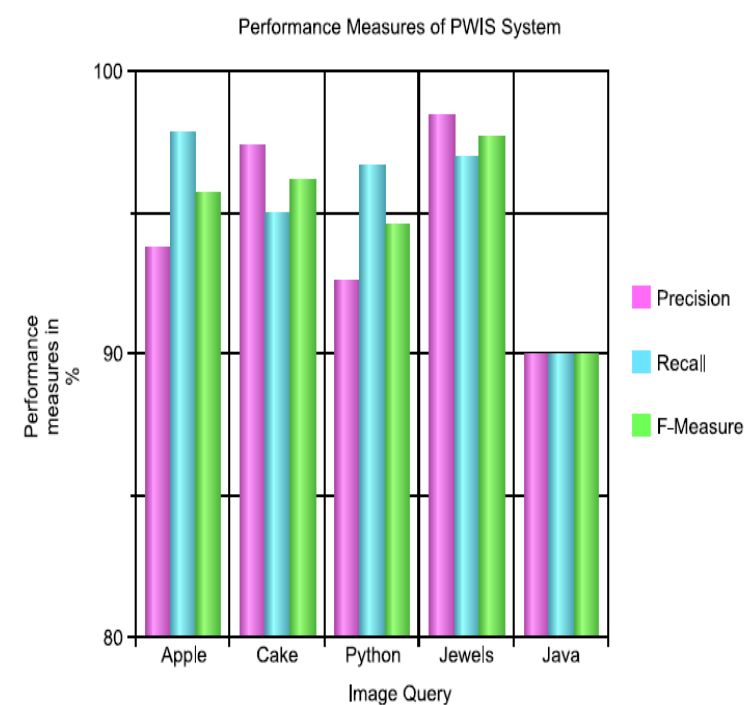

Fig. 4. Performance measures of PWIS

The Fig. 6 gives the comparison of averaged top $\mathrm{m}$ precision of the proposed PWIS system with the existing methods. The image re-ranking by the Pseudo Relevance Feedback [PRF] approach proposed in [19]. It is used for top-ranked images as positive examples to train a one-class SVM. The paper [20] proposed the image re-ranking by the Pseudo Relevance Feedback used for top-ranked images as positive and negative examples to train an SVM. The image re-ranking in transmedia distances presented in [21]. It combined both visual and textual features under the Pseudo Relevance Feedback framework. The ExtBoth $(\mathrm{V}+\mathrm{T})$ approach based on initial image re-ranking and expanding image pool and positive image examples through keyword expansion and image clustering. The proposed PWIS algorithm outperforms better results compared to existing methods, particularly when $\mathrm{m}$ is large.

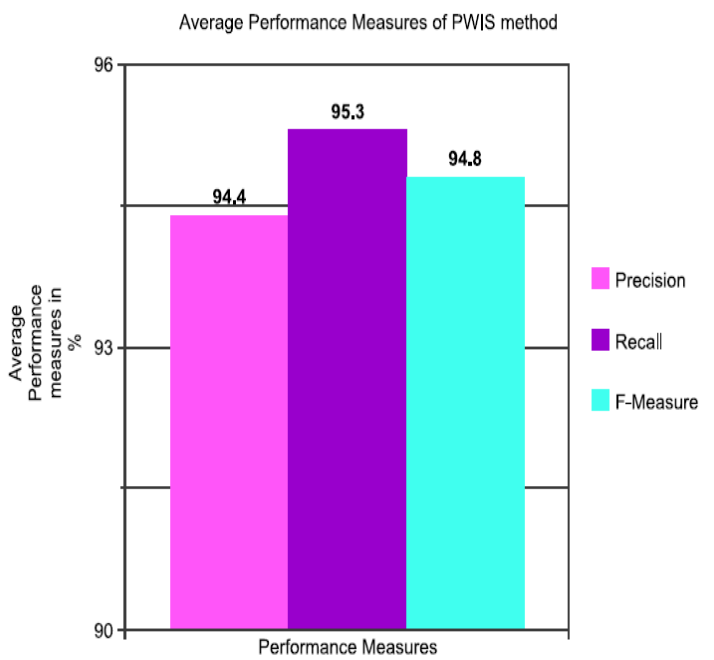

Fig. 5. Average Performance measures of PWIS 


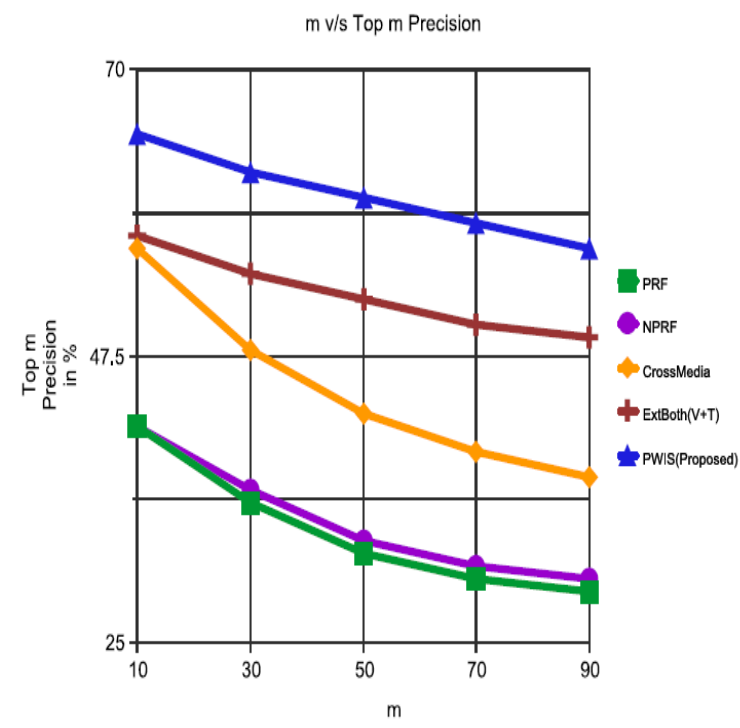

Fig. 6. Comparison of averaged top m precision of PWIS with existing methods.

The drawback of the ExtBoth $(\mathrm{V}+\mathrm{T})$ method is unable to detect the duplication of images in the results. However, the proposed PWIS system recognizes the duplication of images, as images are stored in the database with unique file name. The proposed PWIS can yields better performance compared to the existing methods. The advantage of PWIS system is to detect the duplication of images and by eliminating the duplication of images, system performance is improved.

\section{CONCLUSIONS}

A novel Web-based image search approach which only requires one-click user feedback. Intention specific weight schema is proposed to combine visual features and to compute visual similarity adaptive to query images. Without additional human feedback, textual and visual expansions are integrated to capture user intention. Expanded keywords are used to extend positive example images and enlarge the image pool to include more relevant images. The proposed new image reranking framework consists of multiple steps, which can be improved separately or replaced by other techniques equivalently effective.

Experimental results reveal that the proposed approach is effective to explore the user's interests for image categorization and gives relevant images of user intention by one click. The PWIS system yields the average precision of $94.4 \%$, average recall of $95.3 \%$ and the average F-measure of $94.8 \%$. The proposed PWIS algorithm yields better performance compared to the existing methods, because our system avoids the duplication of the images in the database.

\section{REFERENCES}

[1] K. Yadav and S. Singh, "Improving Web Image Search Re-Ranking using Hybrid Approach", International Journal of Advanced Research in Computer Science and Software Engineering, 2005.

[2] P. Chouragade and P. Chatur, "Visual Re-rank: Applying to Large Scale Image Search as a Soft Computing Approach," International Journal of Management, IT and Engineering, 3(3), 99-115, 2013.

[3] Hsu, W. H., Kennedy, L. S. and Chang, S.F. 2006. Video Search Re-ranking via Information Bottleneck
Principle. In Proceedings of the 14th Annual ACM International Conference on Multimedia, (MULTIMEDIA 06), ACM, 35-44.

[4] Huang, Y., Liu, Q., Zhang, S. and Metaxas, D. N. 2010. Image Retrieval via Probabilistic Hypergraph Ranking, In Proceedings of the IEEE Conference on Computer Vision and Pattern Recognition (CVPR - 2010), 33763383.

[5] Ben-Haim, N., Babenko B. and Belongie, S. 2006. Improving Web-based Image Search via Content Based Clustering, In Proceedings of the SLAM, (New York City).

[6] Fergus, R., Perona P. and Zisserman, A. 2004. A Visual Category Filter for Google Images, In Proceedings of the Computer Vision-ECCV 2004, 242- 256,Springer.

[7] Park, G., Baek, Y. and Lee, H. K. 2006. Web Image Retrieval using Majority-based Ranking Approach, In Proceedings of the Multimedia Tools and Applications, 31(2), 195-219.

[8] Ng, H. T., Leong, M. K., Kan, M. Y. and Ji, D. 2006. Information Retrieval Technology, In Proceedings of the Third Asia Information Retrieval Symposium AIRS 2006, (Singapore).

[9] O. Chum and J. Matas, "Optimal Randomized RANSAC," Journal of IEEE Transactions on Pattern Analysis and Machine Intelligence, 30(8), 1472-1482, 2008.

[10] Ah-Pine, J., Bressan, M., Clinchant, S. Csurka, G. Hoppenot, Y and Renders, J. M. 2009. Crossing Textual and Visual Content in Different Application Scenarios, In Proceedings of the Multimedia Tools and Applications, 42(1), 31-56.

[11] Krapac, J., Allan, M., Verbeek J. and Jurie, F. 2010 Improving Web Image Search Results using QueryRelative Classifiers, In Proceedings of the IEEE Conference on Computer Vision and Pattern Recognition (CVPR- 2010), 1094-1101.

[12] Natsev, A. P., Haubold, A., Tesic, J., Xie, .L. and Yan, R. 2007. Semantic Concept-based Query Expansion and Re-ranking for Multimedia Retrieval, In Proceedings of the 15th International Conference on Multimedia, 9911000, ACM.

[13] Smith, J. R., Naphade M. and Natsev, A. 2003. Multimedia Semantic Indexing using Model Vectors. In Proceedings of the International Conference on Multimedia and Expo (ICME?03),IEEE, 445-448.

[14] Frome, A., Singer, Y., Sha F. and Malik, J. 2007. Learning Globally-Consistent Local Distance Functions for Shape-based Image Retrieval and Classification. In Proceedings of the IEEE 11th International Conference on Computer Vision(ICCV 2007), 1-8.

[15] Lin, Y. Y., Liu, T. L. and Fuh, C.S., 2007. Local Ensemble Kernel Learning for Object Category Recognition. In Proceedings of the IEEE Conference on Computer Vision and Pattern Recognition(CVPR 07), 18.

[16] G. Chechik, V. Sharma, U. Shalit and S. Bengio, "Large Scale Online Learning of Image Similarity through 
Ranking”, Journal of Machine Learning Research, 11, 1109-1135, 2010.

[17] Deng, J., Berg, A. C and Fei-Fei, L. 2011. Hierarchical Semantic Indexing for Large Scale Image Retrieval. In Proceedings of the IEEE Conference on Computer Vision and Pattern Recognition (CVPR-2011), 785-792, IEEE.

[18] Xiaoou Tang, Ke Liu, Jingyu Cui, S, Fang Wen and Xiaogang Wang, "IntentSearch: Capturing User Intention for One-Click Internet Image Search", Journal of IEEE Transactions on Pattern Analysis and Machine Intelligenace, 34(7), July 2012.
[19] He, J., Li, M., Zhang, H., Tong, H. and Zhang, C. 2004. PseudoRelevance Feed Based on Iterative Probabilistic One-Class SVMs in Web Image Retrieval. In Proceedings of the Pacific-Rim Conference Multimedia.

[20] Yan, R., Hauptmann, E. and Jin, R. 2013. Negative Pseudo-Relevance Feedback in Content- Based Video Retrieval. In Proceedings of the 11th ACM International Conference on Multimedia, ACM.

[21] Ah-Pine, J., Bressan, M., Clinchant, S., Csurka, G., Hoppenot, Y. and Renders, J. 2009. Crossing Textual and Visual Content in Different Application Scenarios,Multimedia Tools and Applications, 42, 3156. 